\title{
Estudio de materiales usados en la fabricación de las cerámicas de Fran Ali (Oued Laou, Marruecos)
}

\author{
J. BARRIOS NEIRA ${ }^{1 *}$, J.C. MARTÍN DE LA CRUZ ${ }^{2}$ Y L. MONTEALEGRE CONTRERAS ${ }^{3}$ \\ ${ }^{1}$ Dpto. Química Inorgánica e Ingeniería Química, Edificio Marie Curie. Campus de Rabanales. Universidad. Córdoba 14071. \\ ${ }^{2}$ Depto. Geografía y Ciencias del Territorio. Área de Prehistoria Univ. Córdoba. \\ ${ }^{3}$ Dpto. Ciencias y Recursos A. y F., ETSIAM. Edificio Celestino Mutis. C.Rabanales. Universidad de Córdoba \\ *Tfno. 0034957218620 , E- mail: iq1banej@uco.es
}

\begin{abstract}
Se ha estudiado el comportamiento térmico, la mineralogía, la textura y la microestructura de las materias primas empleadas en la elaboración de las cerámicas de la comunidad alfarera de Fran Ali (Oued Laou, Tetuán, Marruecos). Las rocas originarias de los distintos afloramientos proceden de Unidades Malaguide-Gomárides de la cadena alpina Bético-Rifeña también se estudiaron. Se detalla la técnica empleada por los ceramistas de esta zona para la elaboración de las piezas. Se analizó el agua empleada para la preparación de la pasta. Las rocas originarias son metapelitas o filadios y las materias primas pertenecen a niveles originados por alteración supergénica o exógena (arcillas de tipo cuarzo-illiticas y cloríticas). Las cerámicas estudiadas al microscopio presentan anisotropía.
\end{abstract}

Palabras claves: Cerámicas, materias primas, Marruecos, fabricación, mineralogía, textura.

Study of the raw materials used in the manufacture of ceramics in Fran Ali (Oued Laou, Marruecos)

The thermal behaviour, mineralogy, texture and micro-structure of the original rocks used as raw materials in the manufacture of ceramics of the potter community in Fran Ali (Oued Laou, Tetouan, Morocco) were studied. The original rocks of the alteration deposit are metapelites and altered philites from the Units Malaguide-Gomáride in the Baetic-Rifian mountain system. The techniques used by local potters and the water used to prepare the ceramic bodies were also studied. Original rocks are metapelites or filadios and raw materials belong to levels caused by exogenous or supergene alteration (quartz-illite and chlorite type clays). The ceramics studied by microscopy exhibit anisotropy.

Keywords: Ceramics, raw materials, Morocco, manufacture, mineralogy, texture.

\section{INTRODUCCIÓN}

La razón de este trabajo viene determinada por la iniciativa interdisciplinar del grupo de investigación HUM-262 del plan andaluz de investigación (PAI), al plantear como objetivo de cooperación internacional al desarrollo, la mejora de los comportamientos mecánicos de las producciones cerámicas de la comunidad alfarera de Fran Ali (Marruecos).Se ha comenzado por un estudio geológico de la zona origen de las materias primas empleadas en la elaboración de las cerámicas.

La región de Oued Laou estudiada pertenece geológicamente a Unidades Internas del Arco o cordillera Bético-Rifeña, en concreto a dominios Malaguides-Gomárides, del Paleozoico Superior y Triásico Inferior afectados por un bajo grado de metamorfismo. Esta zona comprende sobretodo el manto superior o más alto de los gomárides (Carbonífero), con facies de metapelitas, formando parte de la secuencia de mantos alóctonos desplazados hacia el W sobre las unidades sébtidas de Beni Bousera (Figura 1.1), (Fig. 1.2). En esta región se observa una inclinación regional hacia el E-NE de las facies metapelíticas (Fig. 1.3).
La deformación causada por la traslación de los mantos alpujárrides y malaguides hacia el NW y de los mantos sébtidos y gomárides hacia el SW (prácticamente simultaneas), ha dado origen a una importante tectonización que ocasiona fuertes deformaciones asociadas de crenulación, kink band, etc. [11], visibles en afloramiento.

La mineralogía encontrada alcanza facies de talco-clorita, siendo las micas y el cuarzo una herencia del sedimento pelítico inicial. La presencia de epidota en las cuñas de kink band indica una fase posterior de epidotización de baja temperatura pero no un aumento metamórfico [7][14].

Los suelos (tipo aridisol y haploxseralf mediterráneo) que cubren de forma discontinua estas zonas son delgados, con índices de enrojecimiento elevados (localmente son rodoxeralf) [12] y apenas se diferencian de las bolsadas de donde se extrae el material para elaborar cerámicas. Los horizontes inferiores Bt de este suelo (arcilla roja) se utilizan como material que aplican en las piezas cerámicas antes de bruñirlas. 


\section{MATERIALES Y TÉCNICAS}

\subsection{Materiales}

En el entorno de FRAN ALI se recogieron las rocas y materias primas, que se emplean en la elaboración de las piezas cerámicas y se realizó un estudio de ellas. Los materiales con los que trabajan pueden clasificarse de calidad algo baja, pero son los que emplean para la elaboración de las cerámicas desde siempre (por encontrarse próximos).

En la zona media de las laderas del valle de Oued Laou, las metapelitas presentan una cubierta de alteración edáfica. En esta posición se observa un cambio de pendiente transversal, donde el espesor de la zona alterada es algo mayor que en el resto y es ahí donde se ubican la mayoría de los yacimientos que se utilizan como canteras a cielo semiabierto para la extracción de la materia prima cerámica. (Figura 1). Los potenciales de extracción de material cerámico son muy bajos y a poca profundidad se alcanza la roca poco alterada

Se han estudiado también piezas cerámicas en los diferentes estadios del proceso de elaboración.

\subsection{Técnicas de análisis}

Mediante el empleo de los siguientes equipos de análisis se han determinado: la composición química, mineralógica, textural y microestructural, así como el comportamiento térmico de los diferentes materiales empleados y de las piezas sometidas a distintos tratamientos de calentamiento.

Para determinar la mineralogía se ha empleado un difractómetro de rayos $X$ Siemens D5000, usando radiación $\mathrm{CuK} \alpha(\lambda=1.54059 \AA)$ con monocromador de grafito.

El estudio microestructural, textural y mineralógico se ha realizado mediante un microscopio petrográfico de polarización Carl Zeiss II POL utilizando láminas delgadas.

El análisis químico semicuantitativo se ha llevado a cabo con un microscopio electrónico de barrido JEOL JSM 6300 acoplado con un analizador EDX (energías dispersivas de rayos X). Cada muestra previamente pulverizada en mortero de ágata, se compacto para obtener una pastilla y se realizaron seis medidas en diferentes zonas de la pieza, para obtener un valor medio de cada una de ellas.

Para el ATD y ATG se empleo un equipo Setsys Evolution 16/18 de Setaram.

La determinación del $\mathrm{pH}$ se realizó mediante un pHmetro "Crison" modelo Digit 2001,

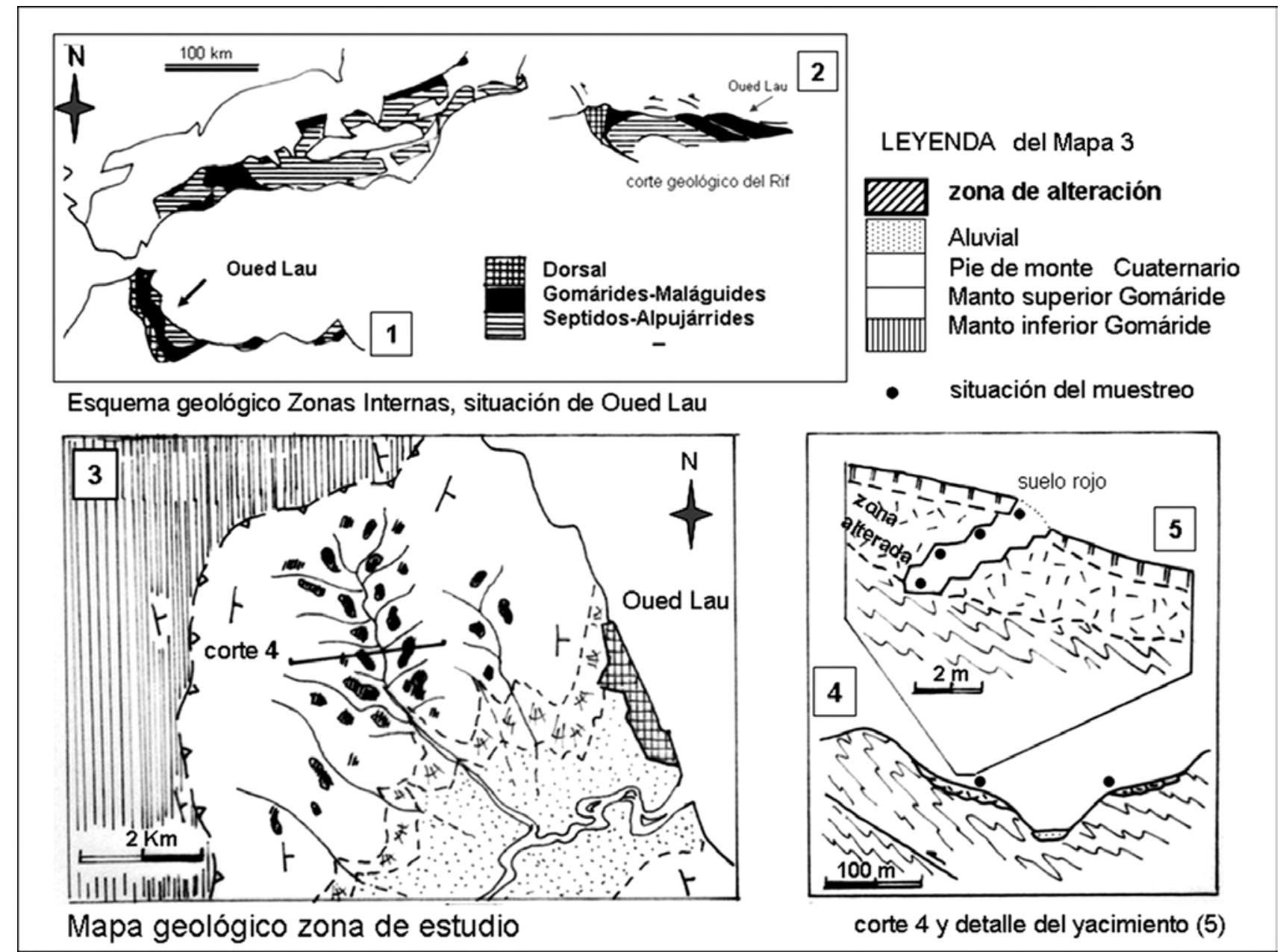

Figura 1. Mapa de situación geológica de la zona estudiada. En 1 se incluye la situación general dentro del Arco Betico-rifeño, basado en Sanz de Galdeano 1992). 2 es un corte geológico modificado por uno de los autores (LMC). 3 es el mapa geológico, cuyos cortes detallados se muestran en 4 y 5 . 
La conductividad eléctrica se midió con un Conductivímetro "Crison" modelo CM 2200

El residuo seco incluye tanto los sólidos orgánicos como los inorgánicos presentes en la muestra que se secó en estufa a $180^{\circ} \mathrm{C}$ hasta peso constante

Los iones $\mathrm{Cu}^{+}, \mathrm{K}^{+}, \mathrm{Mg}^{2+}, \mathrm{Na}^{+}$y $\mathrm{Ca}^{2+}$, se determinaron por Espectroscopía de Absorción Atómica con llama empleando un espectrómetro de Absorción Atómica modelo AAnalyst 100/300 de Perkin Elmer.

La determinación de $\mathrm{NO}_{3}^{-}$se realizó mediante un espectrofotómetro Beckmam DU640. $\mathrm{Los} \mathrm{SO}_{4}{ }_{4}^{2-}$ se determinaron mediante un turbidímetro Hanna Instruments LP2000. Se empleo un Cromatógrafo Iónico DX-500 (DIONEX) para la determinación de $\mathrm{HCO}_{3}{ }^{-}, \mathrm{Cl}$ - $\mathrm{F}^{-}$y $\mathrm{Br}$. Para el análisis de estos aniones se han seguido los métodos estándar descritos por APHA [1]

\subsection{Técnica de preparación (piezas cerámicas)}

Las mujeres realizan de forma manual la extracción de las materias primas en laderas con pendiente próxima a $30^{\circ}$ en afloramientos cercanos al lugar donde se ubica la comunidad alfarera. Con azadas abren un frente de mina que profundizan hasta encontrar la bolsa de alteración y en ella trabajan en sentido lateral (Fig. 1.5). La profundidad en la excavación puede alcanzar unos $3 \mathrm{~m}$ y el frente hasta $5 \mathrm{~m}$.

Para la preparación de la pasta cerámica, toman distintas cantidades de cada una de las materias primas que van a emplear. Las colocan y mezclan sobre el suelo, las cubren con tela de saco y mediante golpes con un palo las trituran. Esta mezcla de materiales triturados después de pasarla por una criba (valor medio de luz de malla de 2,61 mm), es la que van a emplear para la elaboración de las pastas.

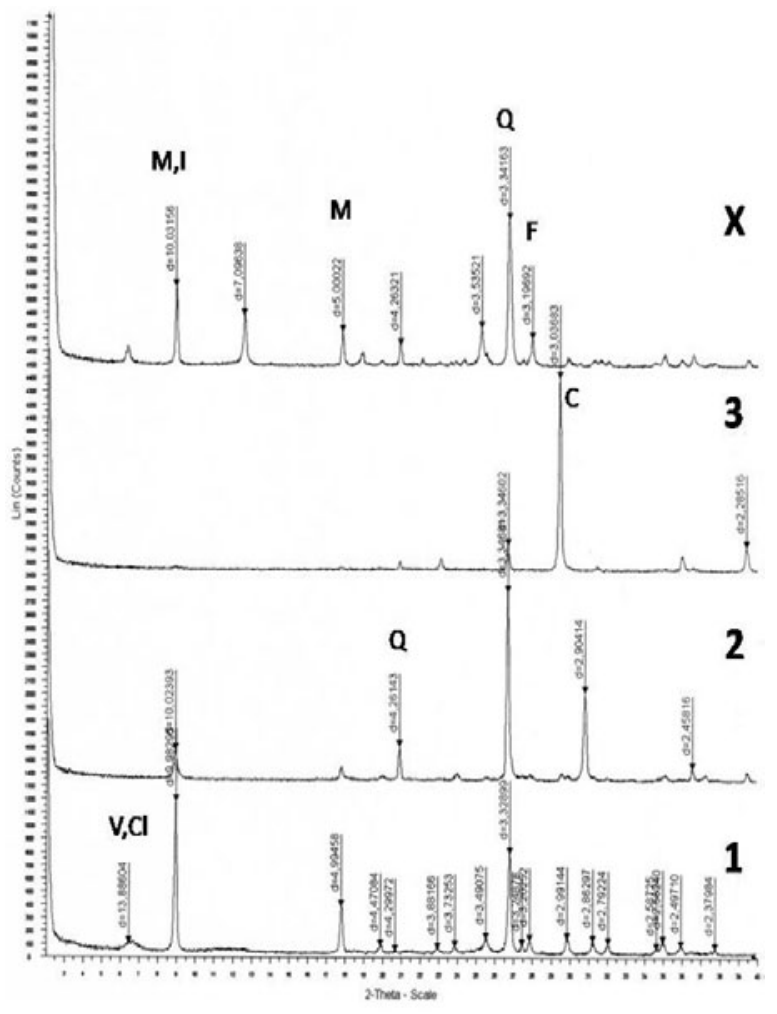

Fig.2. Difractogramas de rayos $\mathrm{X}$ de las rocas 1, 2, 3 y X.
A una cantidad manejable de la mezcla cribada le van añadiendo agua poco a poco hasta que adquiere la plasticidad deseada y comienzan el trabajo de amasado manual de la pasta. Ya amasada se coloca la bola sobre una placa cerámica circular (apoyada sobre una base) que giran manualmente y van dándole forma a la pieza. Terminado el modelado se las deja secar al aire, para la pérdida del agua absorbida y aumento de la manejabilidad. Las cerámicas secas al aire se cubren con arcilla roja y se bruñen con un guijarro.

Se las introduce al horno para una primera cocción a unos $300^{\circ} \mathrm{C}$ que se acaba de llenar con ramaje y se le hace arder. Finalizada esta primera cocción (pérdida del agua de imbibición y coordinación) se deja enfriar el horno antes de sacar las cerámicas. Las que presentan grietas son retiradas $(15 \%)$ y sumergidas en agua para poder reutilizar la pasta. Las piezas en buen estado se introducen de nuevo al horno y se las somete a un nuevo calentamiento con troncos de madera (más compacta), que al arder hace que en el horno se alcancen más altas temperaturas (superior a $\left.600^{\circ} \mathrm{C}\right)$.

\section{RESULTADOS Y DISCUSIÓN}

Las metapelitas estudiadas están expuestas en superficie y presentan un color de alteración debido a minerales pigmentantes (oxihidróxidos de hierro) que acompañan a las arcillas esencialmente illíticas. En la Fig. 1.4 puede verse el perfil transversal de uno de los afloramientos.

El material empleado por los ceramistas, presenta un aspecto masivo, con abundancia de clastos rocosos alterados, y horizontes apenas diferenciados.

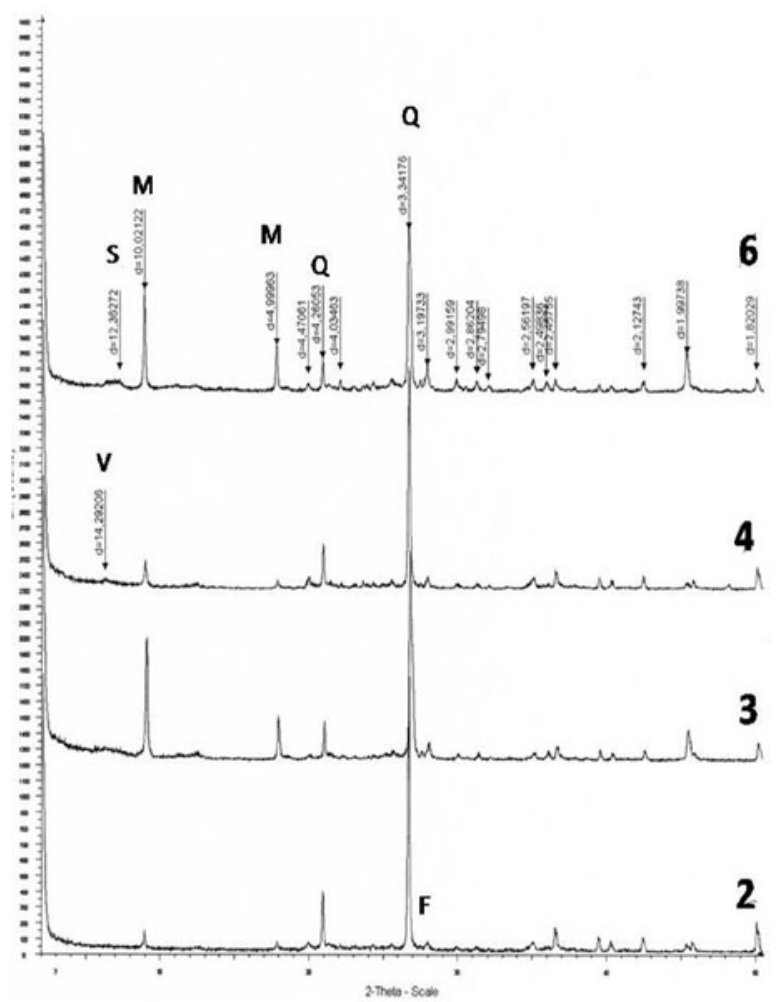

Fig.3. Difractogramas de rayos $\mathrm{X}$ de las materias primas empleadas en la elaboración de la pasta cerámica. 
La forma general de los cuerpos de alteración (y por tanto de los yacimientos) es de lentejones, aunque localmente aparecen en bolsadas no isométricas, de donde se extrae el material para elaborar las cerámicas.

Las manchas de lavado son debidas a procesos de alteración de las arcillas e indican cierto nivel de hidromorfismo, consecuencia posiblemente de una etapa anterior de mayor pluviosidad.

El color de los distintos materiales empleados es según las Tablas Munsell:

- Arcilla roja: manchas 1.5 a $2.5 \mathrm{YR} 6 / 6$,

- Filita sin alterar: amarillo verdoso 5Y7/2.

- Esquisto verde dorado o amarillento sin alterar: 10YRa 2.5Y con 6/4.

- Pasta cerámica: pardo amarillenta de 6 a 7.5 YR $7 / 4$ a $7 / 6$.

- $\quad$ Plato segunda cocción: 3YR5.5/7 a 6.5YR7/6.

- Plato (en el corte): de rojizo 5YR6.5/4 a más pardo 10YR6.5/4.

Los colores verdosos y grises indican poca alteración ya que son propios de las metapelitas. La coloración amarilla puede justificarse por la presencia de pequeñas cantidades $(<1 \%)$ de gohetita que no son detectables mediante difracción de rayos $X$, que señalan inicio de la alteración. La liberación de hematites (por intensa alteración) viene marcada por un
TABla I. DATOS DE ANÁlisis DEL AGUA.

\begin{tabular}{|c|c|}
\hline \multicolumn{2}{|c|}{ 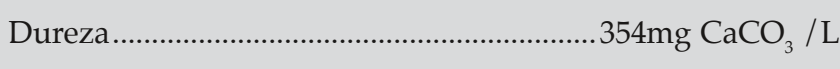 } \\
\hline \multicolumn{2}{|c|}{ 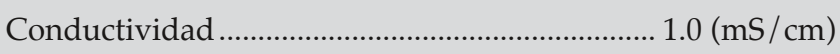 } \\
\hline \multicolumn{2}{|l|}{ Residuo seco $\left(\right.$ a $\left.180^{\circ} \mathrm{C}\right) \ldots$} \\
\hline 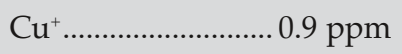 & .........135.7 ppm \\
\hline $\mathrm{K}^{+} \ldots \ldots \ldots \ldots \ldots \ldots \ldots$ & ........... 70.2 “ \\
\hline 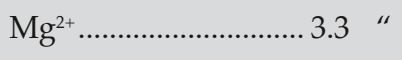 & 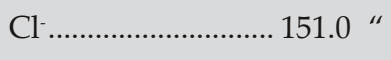 \\
\hline $\mathrm{F}^{-}$ & 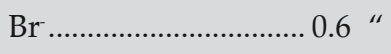 \\
\hline $\mathrm{HCO}_{3}{ }^{-} \ldots \ldots \ldots \ldots \ldots \ldots \ldots . . . . . . . . . .310 .0$ & $\mathrm{SO}_{4}{ }^{2-\ldots \ldots \ldots \ldots \ldots \ldots . . . . . . . . . . . . . . . . . . ~} 70.8$ \\
\hline 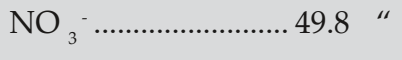 & \\
\hline
\end{tabular}

índice de enrojecimiento que es más acusado en el caso del suelo (arcilla roja).

Los resultados del análisis del agua empleada en la preparación de la pasta cerámica y de la arcilla roja se muestran en la Tabla I. El agua empleada en la elaboración de la pasta cerámica (procede de un acuífero contaminado) presentaba $\mathrm{pH}$ 10. El dato de la conductividad parece indicar un contenido normal de sales. Según los datos obtenidos puede decirse que presenta bastante dureza (a partir de 200ppm se consideran aguas duras). Este tipo de agua básica podría influir en la solubilidad de los óxidos.

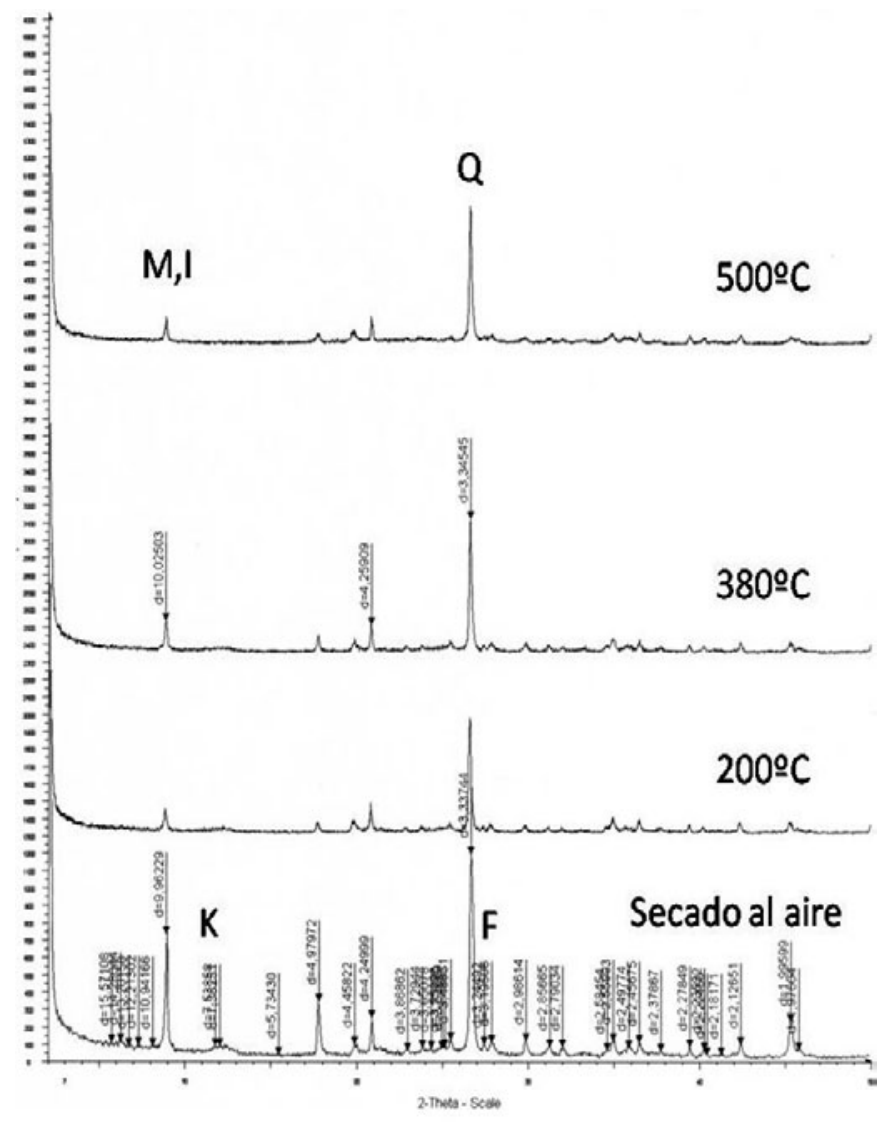

Fig 4. Difractogramas de rayos $\mathrm{X}$ de la arcilla roja: seca al aire, 200, 380 y $500^{\circ} \mathrm{C}$.

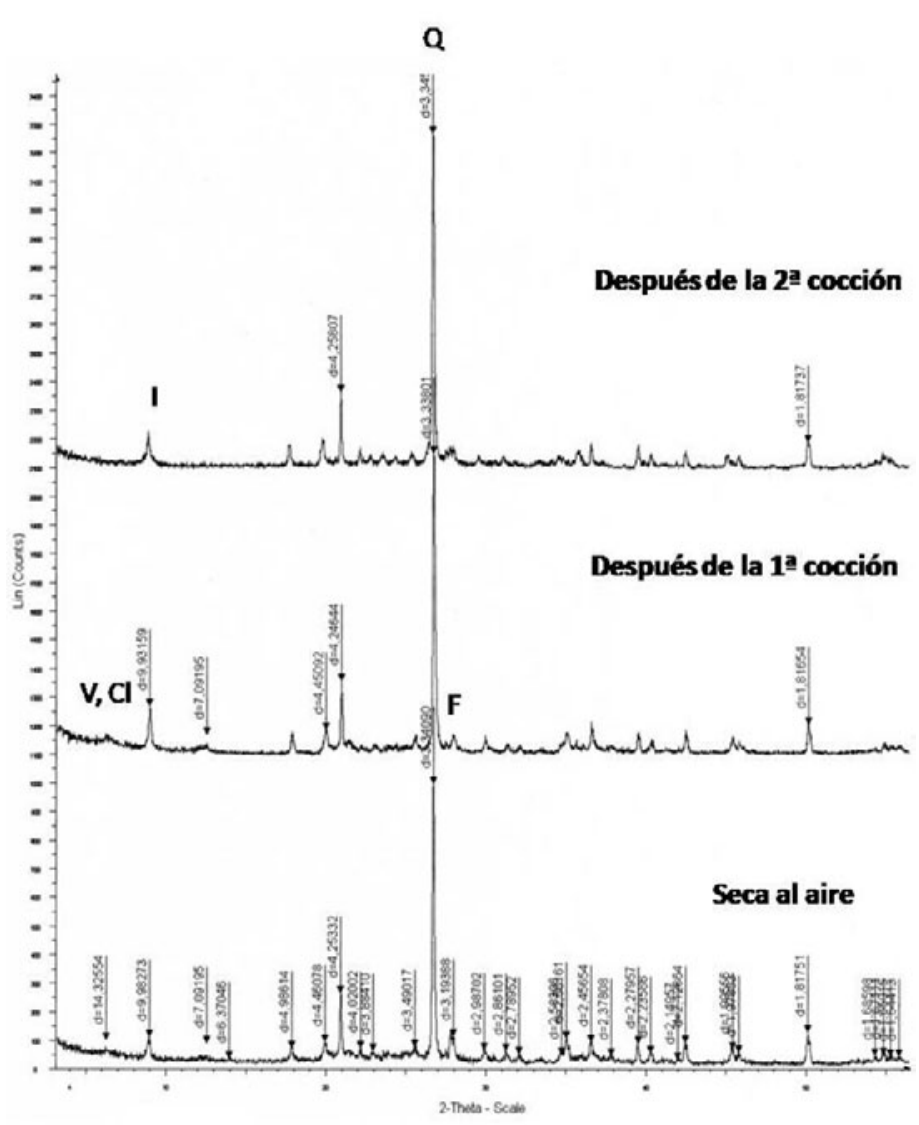

Fig 5. Difractogramas de rayos $X$ de piezas secas al aire y después de la $1^{\mathrm{a}}$ y $2^{\mathrm{a}}$ cocción. 
La Tabla II muestra los datos obtenidos mediante EDX de las materias primas, arcilla roja y plato (seco al aire y después de la segunda cocción) que se expresan en porcentaje de óxidos.

Teniendo en cuenta el posterior estudio mineralógico, los datos encontrados aparecen concordantes con la mineralogía encontrada.

Los valores de los óxidos de $\mathrm{Na}$ y Ca parecen estar en proporción inferior a los valores teóricos, que suelen estar presentes en las rocas pelíticas y sus derivados de alteración, así como en los materiales de extracción y las cerámicas elaboradas con ellos. Son cationes presentes en feldespatos (plagioclasas) y en calcita.

La sílice se distribuye entre el cuarzo y los demás silicatos: feldespatos, micas (Si-Al-Fe-Ti/Mg-K) y talcos (que adscribe el par Si-Mg). El $\mathrm{Mg}$ es un elemento poco abundante, al igual que $\mathrm{Ca}-\mathrm{Na}$, por lo que solo se puede adscribir al talco (escaso) que puede verse en lámina delgada tanto en rocas como en cerámicas. Los elementos Si-Al-K intervienen en los feldespatos y micas. Las micas detectadas (biotita, moscovita y sericita) se llevan gran parte del $\mathrm{Al}$ y $\mathrm{K}$. La presencia de $\mathrm{P}_{2} \mathrm{O}_{5}$ y $\mathrm{SO}_{4}{ }^{2-}$ puede deberse a yeso, materia orgánica o apatito.

Mediante análisis semicuantitativo de los difractogramas de rayos $X$ de las pastas cerámicas se han determinado los valores medios de los minerales hallados en la composición de las pastas cerámicas: $20 \%$ micas, $15 \%$ filosilicatos (vermiculitas, esmectitas, cloritas, kaolinitas), 49\% cuarzo, 2\% calcita (dolomita), $14 \%$ feldespatos.

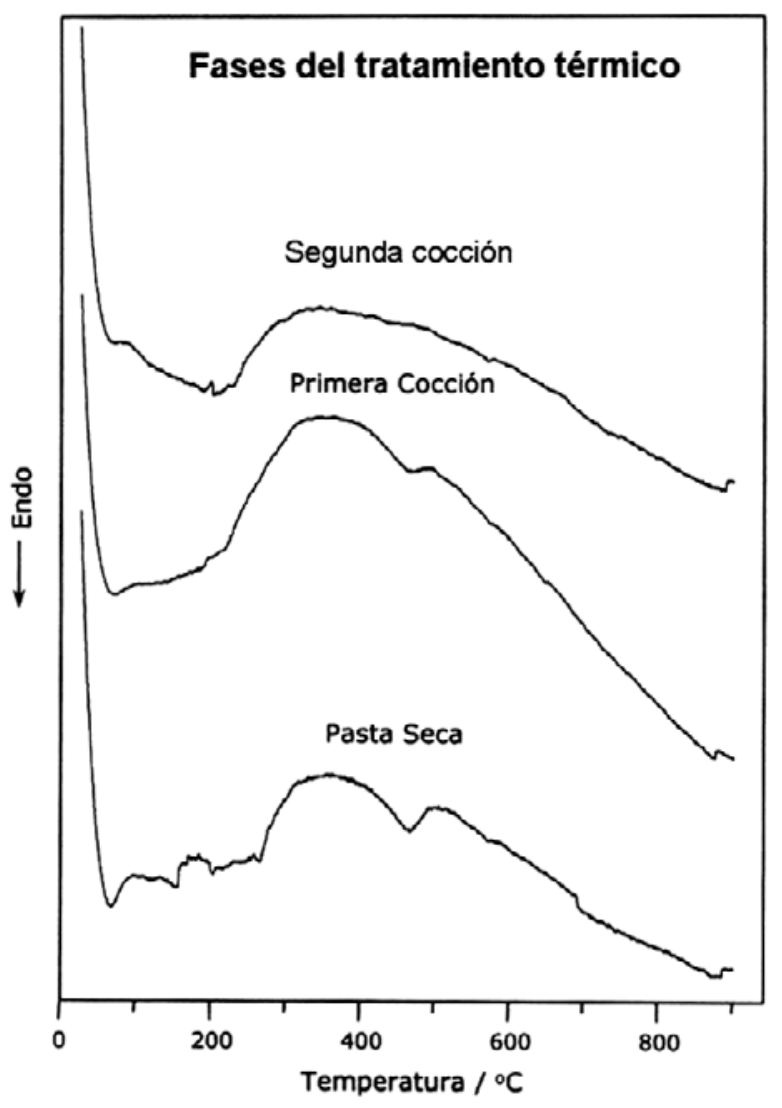

Fig 6. Termogramas de ATD de pasta seca al aire y después de la $1^{a}$ y segunda cocción.

TABla II. DATOS DE \% DE ÓXIDOS POR EDX.(ENERGía DisPERSIVA DE RAYOS X).

\begin{tabular}{|c|c|c|c|c|c|c|c|}
\hline Óxidos & Secas al aire $\%$ & Plato cocido \% & Arcilla roja $\%$ & Material $02, \%$ & Material $03, \%$ & Material 04, \% & Material 06, \% \\
\hline $\mathrm{Na}_{2} \mathrm{O}$ & 0.8 & 1.0 & 0.5 & 1.2 & 1.0 & 0.7 & 1.9 \\
\hline $\mathrm{MgO}$ & 1.1 & 1.3 & 0.9 & 0.8 & 1.4 & 1.2 & 1.5 \\
\hline $\mathrm{Al}_{2} \mathrm{O}_{3}$ & 26.4 & 24.9 & 26.2 & 19.3 & 22.8 & 23.9 & 24.45 \\
\hline $\mathrm{SiO}_{2}$ & 59.2 & 59.4 & 56.4 & 67.6 & 61.1 & 62.1 & 57.8 \\
\hline $\mathrm{P}_{2} \mathrm{O}_{5}$ & 0.1 & 0.2 & 0.1 & 0.04 & 0.1 & 0.1 & 0.1 \\
\hline $\mathrm{SO}_{3}$ & 0.1 & 0.02 & 0.05 & 0.1 & 0.1 & 0.05 & 0.05 \\
\hline $\mathrm{Cl}^{-}$ & 0.1 & 0.03 & -- & 0.5 & 0.1 & 0.02 & 0.1 \\
\hline $\mathrm{K}_{2} \mathrm{O}$ & 3.1 & 3.6 & 4.2 & 2.1 & 3.1 & 2.8 & 4.1 \\
\hline $\mathrm{CaO}$ & 0.2 & 0.3 & 0.05 & 0.02 & 0.2 & 0.1 & 0.1 \\
\hline $\mathrm{TiO}_{2}$ & 0.9 & 0.9 & 1.15 & 0.8 & 1.0 & 0.9 & 1.5 \\
\hline $\mathrm{Fe}_{2} \mathrm{O}_{3}$ & 7.9 & 8.4 & 10.5 & 7.5 & 9.0 & 8.1 & 8.6 \\
\hline $\mathrm{Rb}_{2} \mathrm{O}$ & 0.01 & 0.02 & 0.02 & 0.01 & 0.02 & 0.01 & 0.02 \\
\hline $\mathrm{SrO}$ & -- & 0.01 & .006 & 0.005 & 0.005 & 0.01 & 0.01 \\
\hline $\mathrm{ZrO}_{2}$ & 0.02 & 0.02 & 0.02 & 0.04 & 0.02 & 0.03 & 0.02 \\
\hline $\begin{array}{c}\text { Trazas: } \mathrm{MnO} / \\
\mathrm{Cr}_{2} \mathrm{O}_{3} / \mathrm{BaO} / \\
\mathrm{ZnO} /\end{array}$ & $\begin{array}{l}0.06 / 0.04 / \\
0.01 / 0.01 /\end{array}$ & $--/--/ 0.02 / 0.01 /$ & $--/ 0.04 / 0.03 /--/$ & /-- /--/ 0.03/--/ & $\begin{array}{c}0.04 /--/ \\
0.04 / 0.01 /\end{array}$ & $--/--/ 0.03 /--/$ & $\begin{array}{c}0.07 /--/ \\
0.03 / 0.05 /\end{array}$ \\
\hline
\end{tabular}


Los datos de ATG (Fig. 7) permiten conocer la pérdida de peso determinada a $900^{\circ} \mathrm{C}$ en un $11 \%$ en el caso de las cerámicas secas al aire y de un $1.7 \%$ para aquellas que previamente habían sido sometidas a la $2^{a}$ cocción.

Los difractogramas de rayos $\mathrm{X}$ del material estudiado se muestran en las Figs. 2, 3, 4 y 5.

En la Fig. 2 pueden verse los difractogramas de rayos $X$ [5] de las rocas estudiadas del entorno del lugar de elaboración de las cerámicas (origen de los materias primas empleadas). En ellos puede verse la presencia de cuarzo(Q), micas $(\mathrm{M})$, filosilicatos (Fil), cloritas ( $\mathrm{Cl})$, feldespatos $(\mathrm{F})$, kaolinita $(\mathrm{K})$, en rocas como M-3 presencia de calcita (C) y en M-2 dolomita ferrosa (D). Los minerales que están presentes en porcentajes muy bajos, no se detectan por difracción de rayos X.

La Fig. 3 presenta los difractogramas de las materias primas empleadas para la elaboración de las cerámicas y como era de esperar muestran una mineralogía equivalente a la de las rocas de origen.

La Fig. 4 muestra los difractogramas de rayos $X$ de la arcilla roja (empleada como engobe antes de bruñir la cerámica) después de ser tratada a distintas temperaturas: temperatura ambiente, 200, 380 y $500^{\circ} \mathrm{C}$. La mineralogía observada es: cuarzo, feldespatos, micas y otros filosilicatos. La Foto 6 muestra el aspecto microscópico de dicha arcilla roja (materia prima procedente del suelo)

Los difractogramas de rayos $\mathrm{X}$ de las cerámicas se muestran en la Fig. 5: (a) secas al aire, (b) después de la primera cocción y (c) después de la segunda cocción.

El pico a $7.1 \AA$ correspondiente a la kaolinita puede verse en la pieza seca al aire y también después de la primera cocción, pero ha desaparecido después de la segunda cocción (alrededor de $600^{\circ} \mathrm{C}$ ). Este hecho puede atribuirse a la consiguiente transformación de dicho filosilicato a metakaolinita (según otros a sílice y alúmina amorfa)

Una simple inmersión en agua de las piezas rotas o agrietadas después de la primera cocción permite la reutilización de su masa (recupera la plasticidad) para nuevas piezas, hecho que permite suponer que el calentamiento al que se habían sometido no ha causado trasformación irreversible de ninguna de las materias primas que las componen.

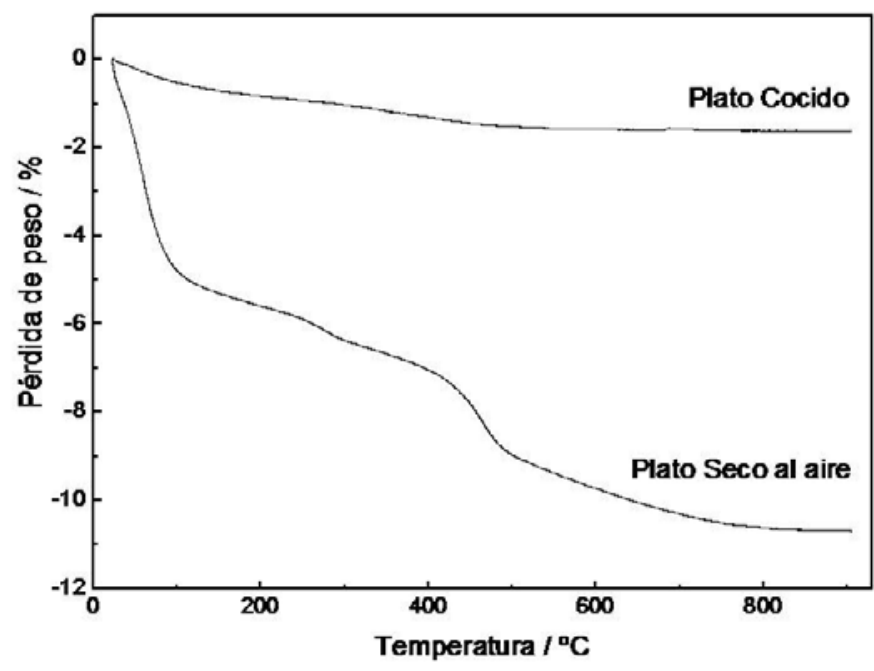

Fig.7. ATG de platos seco al aire y después de la segunda cocción

En la Figura 6 se muestran las curvas correspondientes al análisis térmico diferencial de las cerámicas: a) secas al aire, b) después de la primera cocción y c) después de la segunda cocción. Observando las curvas de ATD [8] de las cerámicas se observan los picos endotérmicos debidos a las pérdidas del agua de imbibición y de coordinación. La desaparición del pico endotérmico hacia $500^{\circ} \mathrm{C}$ después de la segunda cocción puede deberse a la deshidróxilación de la kaolinita. Las piezas rotas después de la segunda cocción sólo pueden recuperarse triturándolas y añadiéndolas a la nueva pasta cerámica como chamota.

La Tabla III muestra el estudio microscópico realizado a las rocas mediante lámina delgada [10],[15]. Todas las rocas estudiadas presentan un cierto grado de alteración supergénica, que originan minerales de la arcilla, cloritas y óxidos de hierro (hematites y gohetita) [4] (Fotos 1, 2, 3, 4, 5).

Las rocas de la Unidad geológica del manto de Goméride Superior sonrocas metamórficas de bajo grado o de la epizona

TABLA III. TEXTURA Y MINERALOGÍA DE LAS ROCAS MEDIANTE LÁMINA DELGADA.

\begin{tabular}{|c|c|c|c|}
\hline Roca & clasificación & textura & Minerales \\
\hline $\mathrm{M}^{\prime \prime}$ & Metapelita arcillosa & Lepidoblástica & $\begin{array}{l}\text { cuarzo, biotita, feldespatos (ortoclasa } \\
\text { y plagioclasa), talco clorita, moscovita, }\end{array}$ \\
\hline M1 & $\begin{array}{l}\text { Metapelita arcillosa con } \\
\text { deformacion lenticular, }\end{array}$ & $\begin{array}{l}\text { lepidoblástica con kind-band, en lentes } \\
\text { de cuarzo con deformación en cizalla }\end{array}$ & $\begin{array}{l}\text { Biotitas y arcillas. Minoritarios: cuarzo en lente, } \\
\text { cloritas, biotita cloritizada en lente, epidota, talco }\end{array}$ \\
\hline M2 & esquisto filadico o micacita & $\begin{array}{l}\text { lepidoblástica con lentes } \\
\text { y microplie- gues kind-band }\end{array}$ & $\begin{array}{c}\text { moscovita, cuarzo, calcedonia, dolomita-Fe/ } \\
\text { siderita-ankerita), Minoritarios: plagioclasas, } \\
\text { ortoclasas , opacos }\end{array}$ \\
\hline M3 & esquisto marmoreo calcítico, & $\begin{array}{l}\text { lepidoblástica y granoblastica } \\
\text { subpoligonal }\end{array}$ & $\begin{array}{c}\text { Calcita poligonal, accesorios: cuarzo, moscovita, } \\
\text { opacos, clorita, talco, arcillas }\end{array}$ \\
\hline $\mathrm{Mx}$ & metapelita o filita cloritizada & $\begin{array}{l}\text { lepidoblástica con micropliegues } \\
\text { kink-band. }\end{array}$ & $\begin{array}{c}\text { biotita, clorita, cuarzo, accesorios: ortosa, } \\
\text { moscovita, talco }\end{array}$ \\
\hline
\end{tabular}




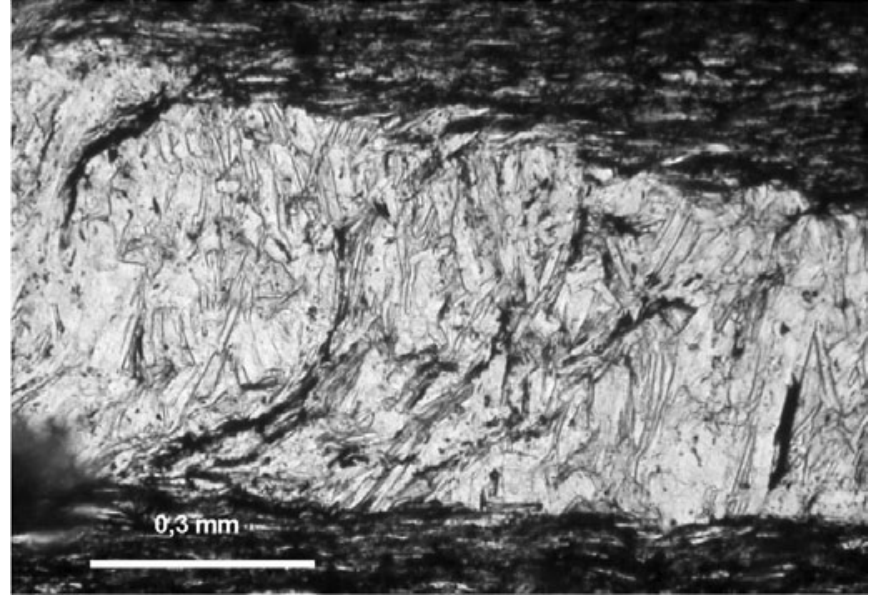

Foto 1. Roca M-1. Metapelita lepidoblástica (sin polarizadores). Detalle de una lente granoblástica de cuarzo con moscovita y epidota (amarilla). Muestra una deformación en la que los cristalitos tienden a ser perpendiculares a la lente. Hay abundancia de arcillas y opacos (lechos lepidoblásticos oscuros).

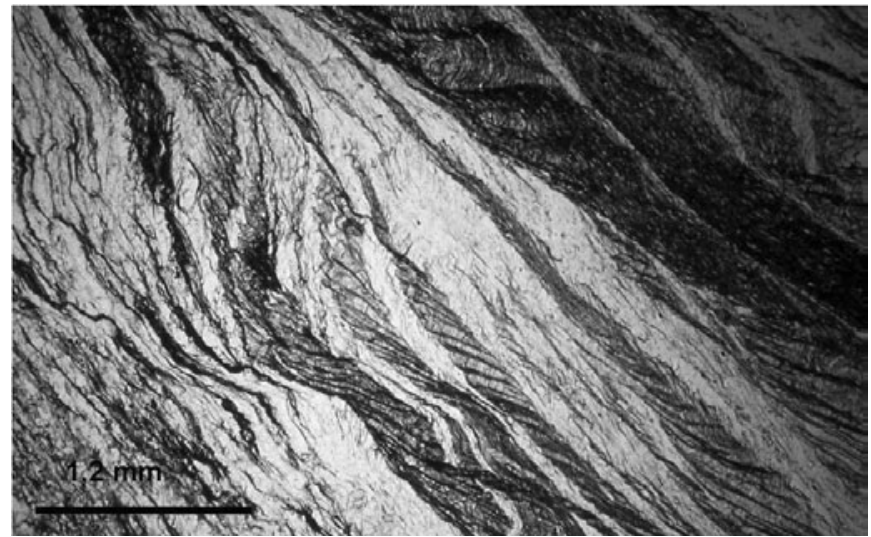

Foto 2. Roca M.2. Metapelita (sin polarizadores). Aspecto general de la metapelita. Alternancia de lechos de cuarzo (color claro) y bandas de micas (oscuras) con deformación por crenulación.

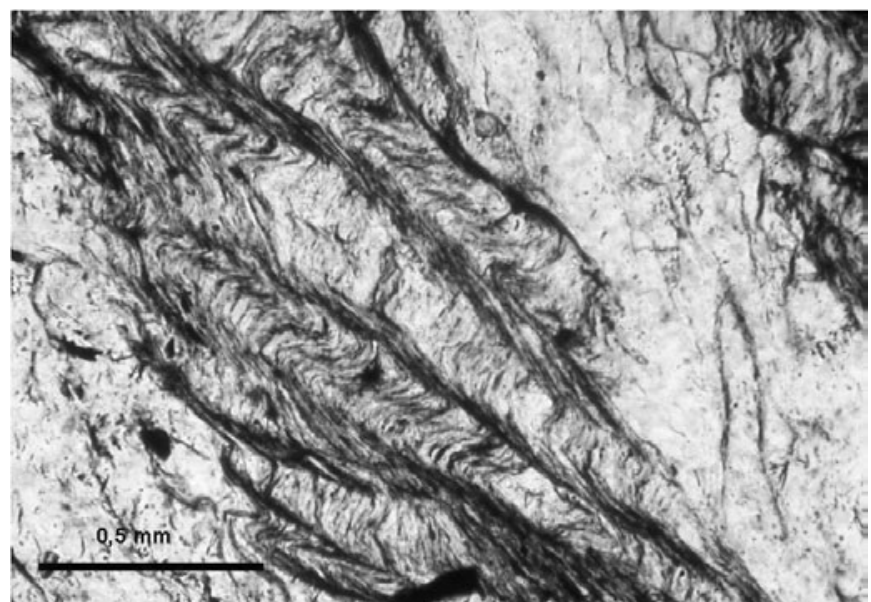

Foto 3. Roca M-2. Detalle de la anterior (sin polarizadores).

Se ven claramente las bandas de micropliegues tipo kink band con más de una fase de deformación y crenulación.

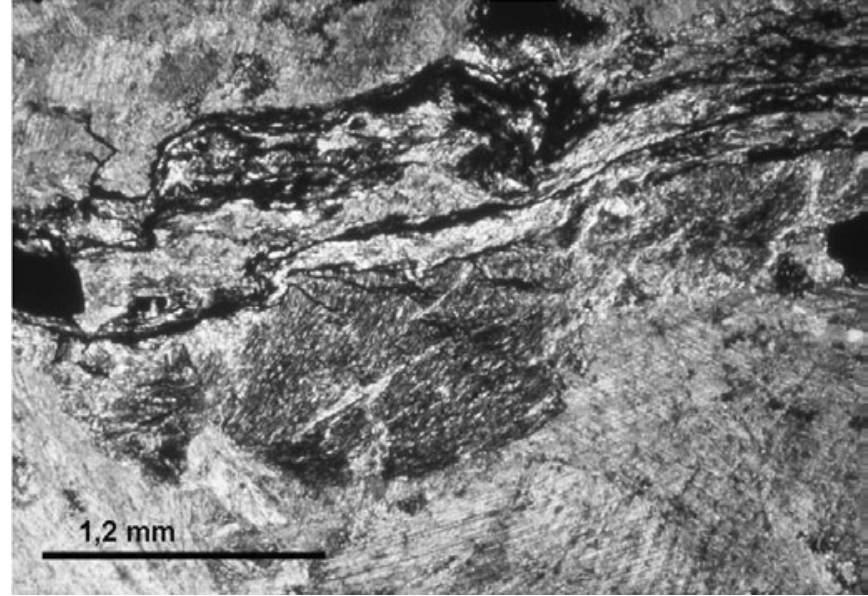

Foto 4. Roca M-3, Esquisto marmóreo (con polarizadores cruzados). Banda de opacos, un núcleo de talco (en el centro), arcillas (amarillas) y predominio de calcita (amarillo sucio) en la foto.

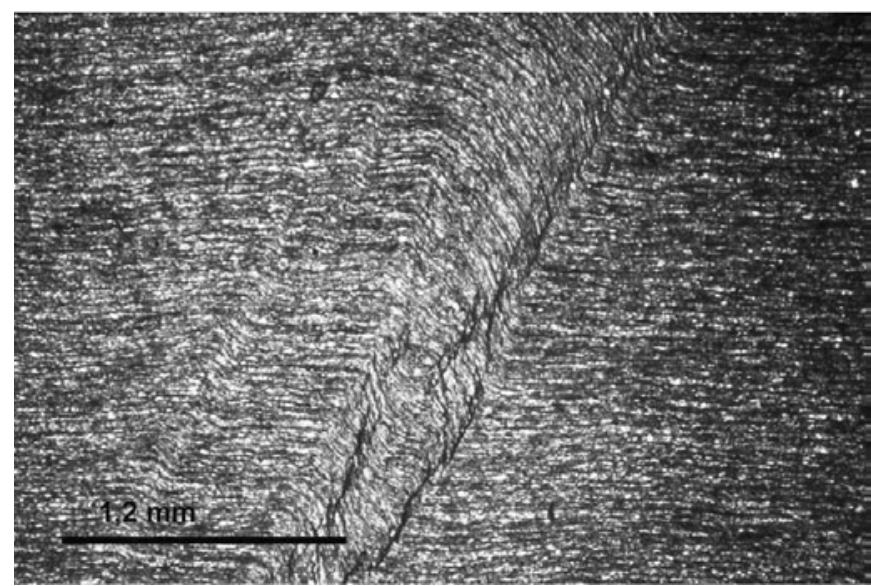

Foto 5. Roca M-x, Metapelita cloritizada (con polarizadores cruzados) Zona con un micro pliegue kink band donde es visible la finura de grano. Presencia de micas, cloritas y cuarzo (mas claro) con orientación lepidoblástica.

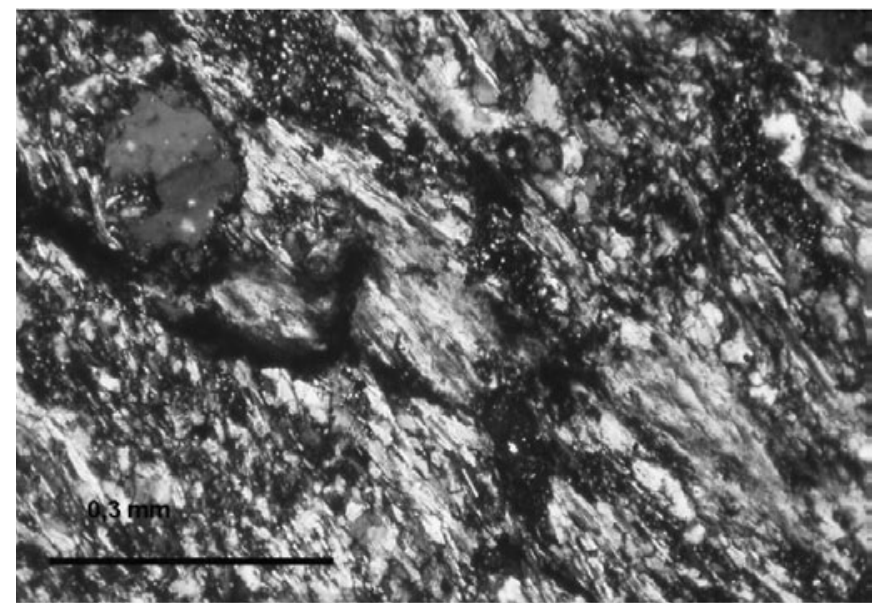

Foto 6. Arcilla roja, pasta de cocción. Se observa la orientación de clastos y granos (cuarzo, micas), y un lentejón de arcilla roja. 
TABLA IV. TEXTURA Y MINERALOGÍA DE CERÁMICAS MEDIANTE LÁMINA DELGADA

\begin{tabular}{|c|c|c|c|c|c|}
\hline muestra & matriz & poros & Matriz/mineral +rocas & minerales & rocas \\
\hline arcilla roja & $\begin{array}{l}\text { Homogénea, } \\
\text { anisótropa } \\
\text { (eskelsépica) }\end{array}$ & $15 \%$ & $35 / 10+55$ & $\begin{array}{c}\text { biotita,cuarzo, } \\
\text { hematites,moscovita, sericita. }\end{array}$ & $\begin{array}{c}\text { clastificadas, } \\
\text { metapelita,filita, cuarcita. }\end{array}$ \\
\hline seco al aire & $\begin{array}{l}\text { Homogénea, } \\
\text { anisotropa* }\end{array}$ & $10 \%$ & $60 / 10+30$ & $\begin{array}{c}\text { Cuarzo,biotita,talco, } \\
\text { hematites,ortoclasa, } \\
\text { plagioclasa,clorita, moscovita. }\end{array}$ & $\begin{array}{l}\text { cuarzo-lutita, } \\
\text { micacita,filita, meta- } \\
\text { cuarzo-arenita. }\end{array}$ \\
\hline $1^{a}$ cocción & $\begin{array}{l}\text { Homogénea, } \\
\text { anisótropa* }\end{array}$ & $10 \%$ & $60 / 10+30$ & $\begin{array}{l}\text { cuarzo,clorita,biotita } \\
\text { hematites,moscovita, } \\
\text { ortoclasa,plagioclasa }\end{array}$ & $\begin{array}{l}\text { cuarzo-lutita, } \\
\text { micacita,filita, meta- } \\
\text { cuarzo-arenita }\end{array}$ \\
\hline $2^{a}$ cocción & $\begin{array}{l}\text { Homogénea, } \\
\text { anisotropa* }\end{array}$ & $<10 \%$ & $60 / 10+30$ & $\begin{array}{l}\text { biotita,hematites, } \\
\text { cuarzo,moscovita, } \\
\text { ortoclasa, plagioclasa. }\end{array}$ & cuarzo-lutita, micacita \\
\hline
\end{tabular}

$\left({ }^{*}\right)$ presentan orientaciones: crystica, eskelsépica, vosépica y masépica (nomenclatura basada en Barrios et al. 2010)

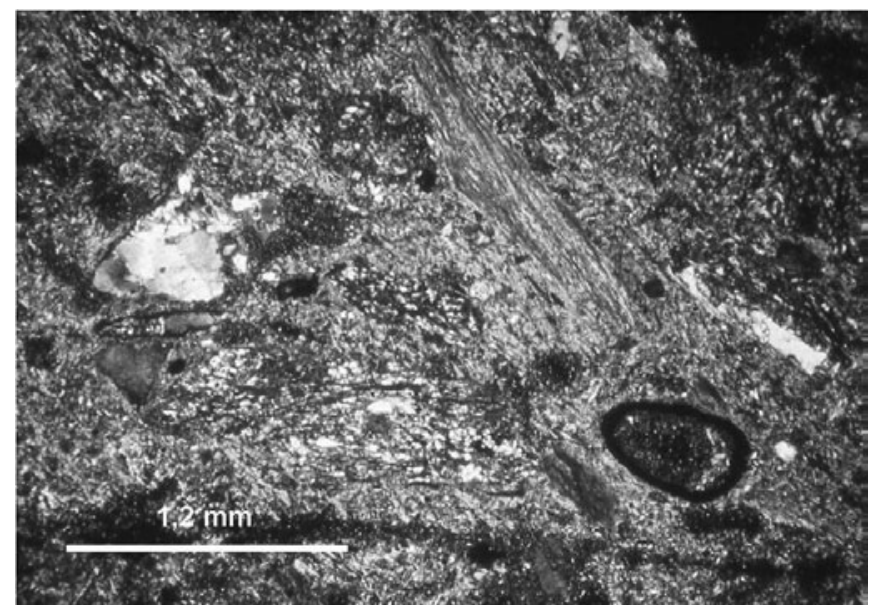

Foto 7. Pasta cerámica arcillosa seca al aire (con polarizadores cruzados). Pasta arcillosa, crístalina con orientación anisótropa: eskélica y masépica. Los clastos rocosos son abundantes y visibles de filitas, metapelitas (con baja alteración) y metapelitas cuarzosas (en el centro y a la izquierda). Restos de vegetal escasamente carbonizado (ovoide oscuro a la derecha)

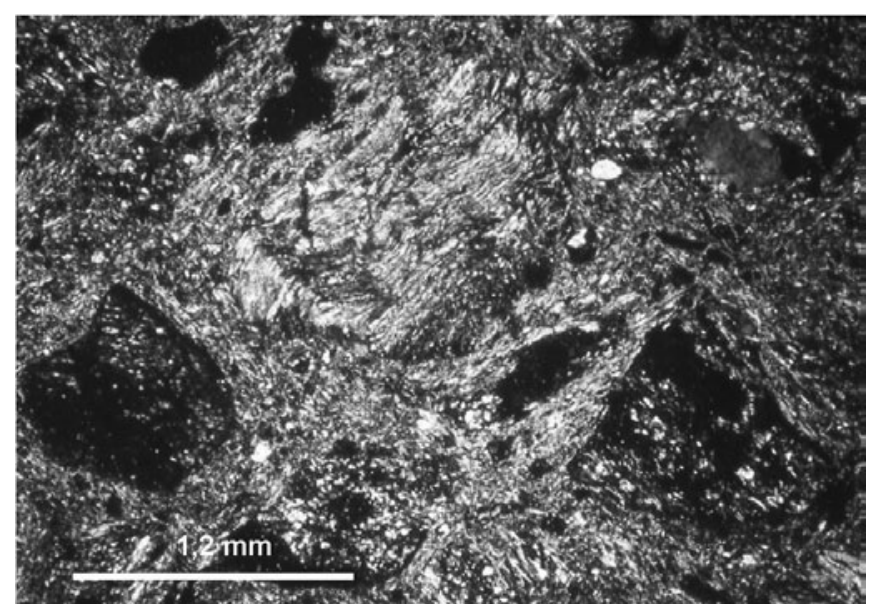

Foto 8. Pasta cerámica después de la $1^{\text {a }}$ cocción (con polarizadores cruzados). Aspecto general. Se ven varios clastos rocosos. Uno de ellos (centro) es una metapelita que presenta deformación y suave alteración rojiza. Otros clastos son mas cuarzosos.

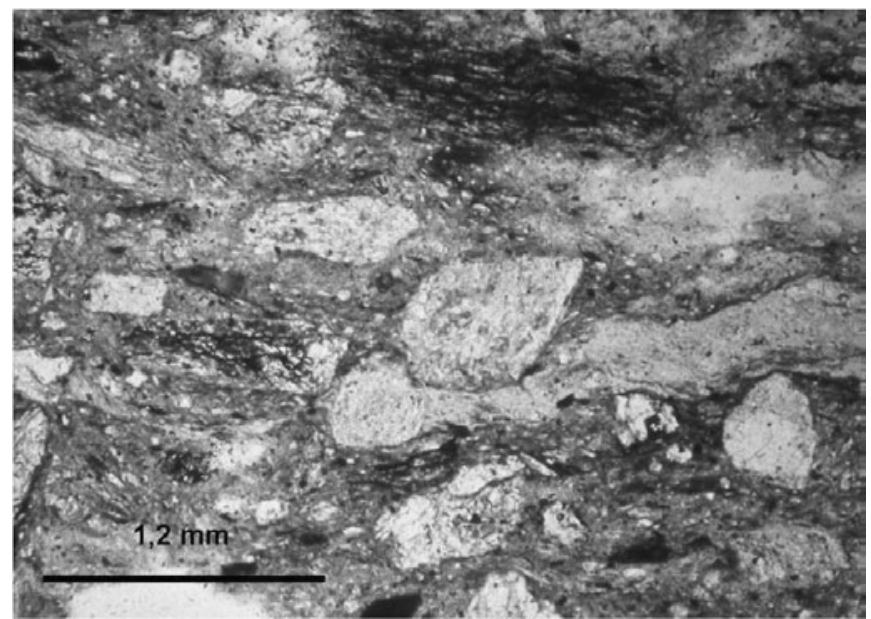

Foto 9. Pasta cerámica final, después de la $2^{\text {a }}$ cocción (sin polarizadores). Abundancia de clastos rocosos. La matriz cementante está fuertemente orientada (anisotropía solo visible bajo polarizadores cruzados)

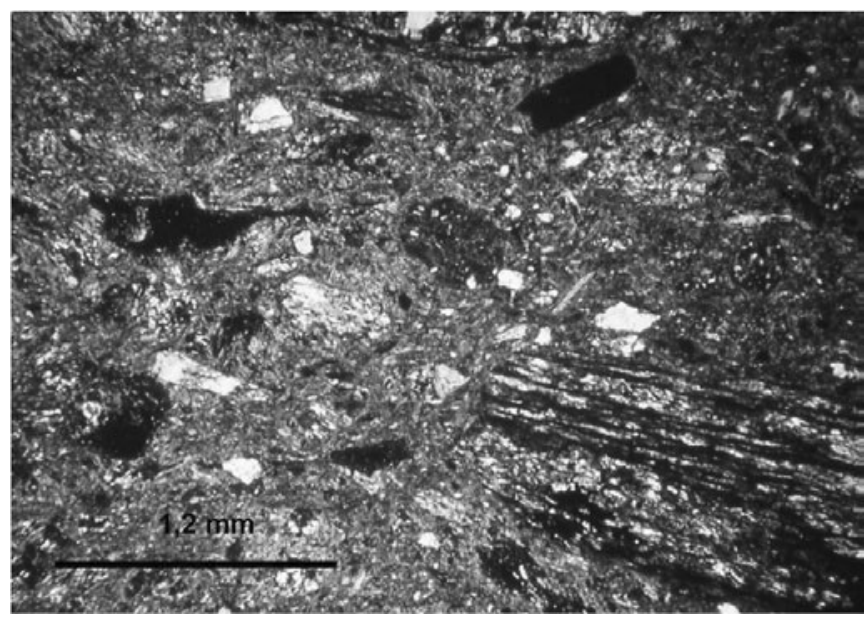

Foto 10. Pasta despues de la $2^{\mathrm{a}}$ cocción (con polarizadores cruzados). Aspecto general de la pasta arcillosa cristalina, totalmente anisótropa, con orientaciones eskelsepicas (alrededor de clastos) y masepica (toda la masa). Los clasots son de metapelitas (cuarzo-lutita), y alguno muestra alteración e impregnación de hematites. 
baja. Se clasifican como metapelitas o filitas (Foto 2) y (Foto 5) y en ellas aparecen facies de cloritas junto a micas (moscovita). Muestran deformaciones características (recristalizaciones micropliegues tipo kink band, etc) que corroboran su origen bajo procesos tectónicos y un metamorfismo regional de bajo grado. En las alineaciones tectónicas (base del manto) pueden aparecer fases de mayor presión (lawsonita-glaucofana), hacia el $\mathrm{N}$ donde se sitúa el límite del manto, pero no en la zona muestreada.

Son en parte rocas cloríticas con mineralogía de micas, cuarzo, cloritas y talco, siendo las cloritas de neo formación posterior. Las texturas presentan orientaciones lepidoblásticas con locales lechos en lente, donde abunda el cuarzo, con texturas granoblásticas locales (Foto 1). Son frecuentes las estructuras de micro-pliegues tipo kink band (Foto 5) y la deformación por crenulación (Foto 2) y (Foto 3) [14]. Tales aspectos estructurales tienen su interés en la facilidad de fragmentación y la consiguiente alterabilidad exógena. Todo ello ha condicionado la existencia de las bolsadas de material alterado utilizadas por las ceramistas.

Las cerámicas tienen una composición de origen inconfundible [2], [3] ya que abundan en ellas clastos rocosos de las citadas filitas, si bien estos muestran la alteración algo más marcada (presencia de hematites) (Tabla IV), (Fotos 7, 8 y 10). Los clastos son muy abundantes, como cabe esperar del sistema de cribado. Los diámetros de los clastos medidos están entre 0,1 a 2,5 mm. En las cerámicas aparece también una pasta fina arcillosa más o menos coloreada.

El aspecto textural de las piezas secas al aire y después de la primera cocción no parece estar afectado, según puede verse al comprobar su semejanza en las correspondientes láminas delgadas (Fotos 6 y 8) con polarizadores cruzados.

Las relaciones texturales [6] de la matriz (pasta fina) de las cerámicas son las esperadas: orientaciones muy marcadas (total anisotropía), especialmente eskélicas (alrededor de los clastos y granos cristalinos) y a veces vosépicas (alrededor de huecos y poros visibles). No hay diferenciaciones de borde-núcleo (Fotos 8 y 10$)$.

No se advierten diferencias claras entre las texturas de las pastas cerámicas: secas al aire y después de la primera y segunda cocción (Fotos 7, 8, 9 y 10). No se ha evidenciado cambio en el porcentaje de porosidad visible al microscopio óptico, aunque es de suponer que dicho cambio debe ocurrir.

La porosidad al agua de las cerámicas después de la $2^{\mathrm{a}}$ cocción es de un $14.5 \%$.

\section{CONCLUSIONES}

- Los materiales empleados para la elaboración de las cerámicas son materiales de alteración procedentes de rocas tipo filitas o metapelitas de bajo grado de metamorfismo.

- En gran parte, las rocas son cloríticas, o cloritizadas, con mineralogía de micas y cuarzo, y en pequeña proporción cloritas y talco.

- Las texturas son orientadas, lepidoblásticas y con locales granoblásticas. Son frecuentes las estructuras de micro pliegues tipo kink band, que delatan la deformación tectónica.
- Los niveles utilizados por los ceramistas son pequeñas zonas de bolsadas y lentejones originados por alteración supergénica o exógena, con enrojecimiento por oxihidróxidos de hierro.

- Los barros son de tipo cuarzo-illitica y cloríticas, y los clastos de filadios o metapelitas.

- El tamaño de los clastos (diámetro entre 0.5 y $2.5 \mathrm{~mm}$ medidos en lámina delgada) y su abundancia son debidos a una deficiente trituración y al sistema de cribado.

- Las relaciones texturales en la matriz de las cerámicas son las esperadas: (a) orientaciones muy marcadas, (b) anisotropía especialmente del tipo eskelsépica (alrededor de los clastos y granos cristalinos) y a veces vosépica (alrededor de huecos y poros visibles), (c) fino manejo por presión durante la elaboración de la pieza.

- La segunda cocción origina la deshidróxilación de la kaolinita y el comienzo de la de otros filosilicatos.

- Las materias primas cerámicas empleadas no son de buena calidad dada su composición y textura, pero son las empleadas desde hace siglos.

\section{REFERENCIAS}

(1) American Public Health Association. Standard Methods for the Examination of Water and Wastewater, 17th ed. Washington, DC, USA. (1989).

(2) J. Barrios Neira, L. Montealegre y L.A. López Palomo. Ceramics of Ategua (Córdoba, Spain): Mineralogical and petrographic study. Applied Clay Science 42, 529-537 (2009).

(3) J. Barrios Neira, L. Montealegre y L.A. López Palomo. Caracterización mineralogica y textural de Cerámicas Tartésicas de Ategua (Córdoba, España). Bol. Soc. Esp. Ceram. V. 49, 5, 361- 371 (2010).

(4) V. Barrón. y L.Montealegre. Iron Oxides and Color of Triassic Sediments: Application of the Kubelka-Munk Theory. Amer. Journ. Science, v. 286: 792-802 (1986).

(5) Brindley \& Brown. Cristal Structures of Clay Minerals and their X-Ray Identification" Ed. Mineralogical Society.(London).(1980).

(6) Bullock, Fedoroff, Jongerius, Stoops, Tursina \& Babel edit. Handbook for Soil Thin Section Description. (Micromorphology of Soils). Waine Research Public. Albrighton, Cambridge Press, (1985).

(7) J. Kornprobst. Manual de Petrología Metamórfica y su contexto geodinámico. Ed. Masson. Barcelona.(1996)

(8) C.A. Jouenne. Traité de Ceramiques et Materiaux Mineraux. Editions Septima. París (1975).

(9) J.Cl. Martín de la Cruz. La comunidad alfarera de Fran Ali. Pub. Univ. Córdoba (2006) 69 páginas.

(10) Melgarejo edits. Atlas de Asociaciones Minerales en Lámina Delgada. Edic. Univ. de Barcelona, 1076 p (1997).

(11) C. Sanz de Galdeano. La Zona Interna Bético-Rifeña. Pub. Univ. de Granada.(1996).

(12) Soil Survey Staff. Keys to Soil Taxonomy. Technical Monograph $\mathrm{M}^{\mathrm{o}} .19$. Blacksburg Fiftj Ed. 541 p (1992).

(13) J.A.Vera. Geología de España. IGME, AGE, MIE, Madrid. (2005)

(14) R.H.Vernon. A Pactical Guide to Rock Microstructure. Cambridge Ed. (2004).

(15) B.W.D.Yardley, W.S.Mackenzie and C.Guilford. Atlas of metamorphics rocks and their textures. Ed. Lougman G.L. Harlow Essex, England. (1990).

Recibido: $07 / 12 / 2010$

Aceptado: 07/10/2011 